\title{
LEVANTAMIENTO, ANÁlisis Y PUBLICACIÓN DE GEOINFORMACIÓN EN UN VISUALIZADOR 3D.
}

\author{
GATHERING, ANALYSIS AND PUBLICATION OF GEOINFORMATION IN A $3 D$
} VISUALIZER.

\section{FABIÁN SANTANDER ${ }^{1}$, VILLIE MOROCHO ${ }^{2}$}

\section{1,2DEPARTAMENTO DE CIENCIAS DE LA COMPUTACIÓN, FACULTAD DE} INGENIERÍA, Universidad de Cuenca. Av. 12 de Abril y Agustín Cueva, Cuenca, Ecuador. 1 fabian. santander@ucuenca.edu.ec,2villie.morocho@ucuenca.edu.ec.

Recibido: 15 de agosto 2018 /Aceptado: 25 de septiembre 2018

\section{RESUMEN}

Los Sistemas de Información Geográfica (GIS) son herramientas para encontrar respuestas de ámbito espacial, que permiten apoyar con las necesidades estratégicas de una entidad. El presente trabajo muestra el resultado de un proyecto SIG sobre el deslizamiento de masas en un territorio, que pretende exponer el resultado de un trabajo mediante el proceso que se ha llevado a cabo en un proyecto enfocado a la vulnerabilidad ante eventos naturales, centrado en los deslizamientos de masa que puede sufrir un territorio determinado a consecuencia de distintos factores. En este documento se va a describir como al utilizar distintas tecnologías se puede obtener información geográfica en tres dimensiones. Partiendo de la participación de un ciudadano común, para la generación de geometrías en dos dimensiones que van a servir para un posterior análisis, que permitirá dar paso al uso de equipos tecnológicos capaces de levantar información con precisión geográfica, para una evaluación detallada de una zona determinada.

Palabras clave: Infraestructura de Datos Espaciales, Visualizador de mapas, 3D, Nube de puntos, SIG.

\begin{abstract}
The Geographic Information Systems (GIS) are tools to find answers of spatial scope, that allow to support with the strategic needs of an entity. The present work shows the result of a GIS project on the landslide in a territory, which aims to expose the result of a work through the process that has been carried out in a project focused on vulnerability to natural events, focused on the landslides that a determined territory can suffer as a result of different factors. In this document we will describe how using different technologies you can obtain geographic information in three dimensions. Starting from the participation of a common citizen, for the generation of geometries in two dimensions that will serve for a later analysis, that will allow to give way to the use of technological equipment able to raise information with geographic precision, for a detailed evaluation of an area determined.
\end{abstract}

Keywords: Spatial Data Infrastructure, Map viewer, 3D, Point cloud, GIS.

\section{INTRODUCCIÓN}

La información geográfica ha revolucionado, y lo sigue haciendo en la manera de representar la superficie terrestre y los objetos situados sobre ella. De tal manera, cada vez es más la información geográfica que es representada digitalmente en distintas formas, ya 
sean en $2 \mathrm{D}, 2.5 \mathrm{D}$ o $3 \mathrm{D}$, todas estas con el fin de ofrecer un mejor análisis y obtener los mejores resultados para la toma de decisiones.

Para justificar la importancia de los Sistemas de Información Geográfica (SIG) y el papel que estos juegan hoy en día, es importante citar el hecho de que aproximadamente un 60 $\%$ de la información que manejamos en cualquier tipo de disciplina esta georreferenciada (Hahmann \& Burghardt, 2012). Es decir, que se trata de información a la cual puede asignarse una posición geográfica, y es por tanto información que viene acompañada de otra información adicional relativa a su localización (Olaya, 2014).

Si bien es probable que este porcentaje de la información georreferenciada ha crecido de manera favorable en comparación de años anteriores, se puede decir que esto ha permitido el desarrollo de múltiples herramientas para hacer uso de toda esa información con el fin de visualizarla y analizarla de distintas maneras. Tal es el caso de un visualizador de nube de puntos geográficos (3D - Tres dimensiones) que ha puesto a disposición la Universidad de Cuenca, con la finalidad de ofrecer una perspectiva adicional a la que ha venido trabajando en los últimos años en el tema de las IDE (Infraestructura de Datos Espaciales).

La implementación de un visualizador de nube de puntos o visualizador 3D, nace de la necesidad de exponer la información geográfica que se ha venido obteniendo gracias a los equipos con los que cuenta la Universidad de Cuenca (Drone eBee y Escáner laser LIDAR). La posibilidad de visualización de una nube de puntos una representación más real de la información obtenida, al contrario que una representación básica en dos dimensiones. Considérese además que la visualización 3D de una nube de puntos es más real para un usuario no familiarizado con mapas vectoriales. El lograr un visualizador de estas características permite aprovechar al máximo la ventaja de los sensores remotos utilizados en el proyecto y que un usuario pueda verlos sin necesidad de una computadora de mayores características más que una conexión a internet.

El presente artículo enmarca la descripción de un conjunto de herramientas y procesos, como parte de un proyecto que busca la manera de relacionar varias tecnologías, con la finalidad de proporcionar información geográfica que pueda estar a disposición en la WEB exhibida en varios formatos dimensionales.

\section{APLICACIÓN MÓVIL}

Los dispositivos móviles inteligentes abarcan una gran cantidad de beneficios haciendo uso de las aplicaciones correspondientes; estas aplicaciones ayudan en la solución de problemas que se pueden presentar en diversos escenarios. Con tal fin la Universidad de Cuenca ha desarrollado una aplicación móvil en el lenguaje de Android, que sirve como un complemento para aportar información geográfica a la plataforma IDE. 
Debido a que Ecuador se encuentra en una zona con diversidad de riesgos naturales que pueden incidir sobre una gran parte de la población, la APP móvil que se ha desarrollado en este proyecto involucra el concepto PPGIS (Public Participation Geographic Information System), este término tiene como finalidad apoyar la participación pública con el objeto de incluir a las poblaciones locales o marginales en los procesos de planificación o decisión (Brown, 2012).

\section{APP MÓVIL Y LA PLATAFORMA IDE UCUENCA}

El diseño del esquema funcional entre la APP móvil y la Plataforma IDE UCuenca, está planteado con cualidades dinámicas, enfocándose principalmente en la emisión de información y la actualización de los elementos que componen la APP móvil mediante la interacción de la Plataforma IDE (Figura 1).

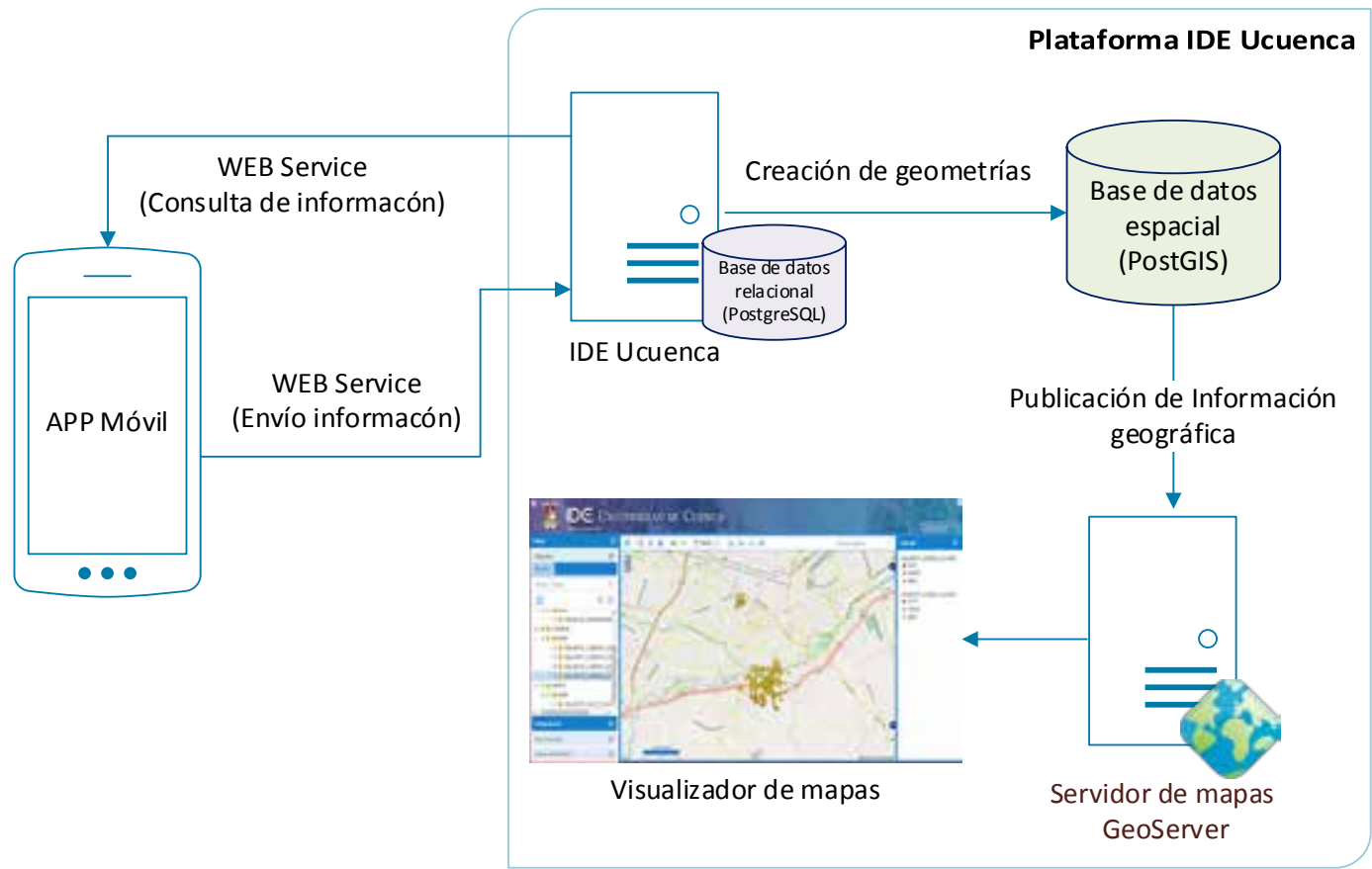

Figura 1. Esquema funcional entre la APP móvil y la Plataforma IDE UCuenca.

Tratándose de un proyecto que busca la participación ciudadana para la generación de información geográfica, la APP móvil está diseña para encuestar al usuario mediante formularios que puedan ser interpretados de una manera intuitiva, asistiendo únicamente con información necesaria al momento de ser presentada, es decir, no todos los parámetros de la encuesta van a estar visibles en un solo formulario, éstos van a visualizarse según la selección que realice el usuario (Figura 2). Esta técnica es conocida como encuesta de tipo árbol, que simplemente es una versión modificada de la técnica de encuesta habitual caracterizada por que las preguntas que se le hacen a cada individuo depende de las respuestas que ha dado con anterioridad, con arreglo a un cuestionario rígido, establecido previamente que define las diferentes rutas posibles (Sequeiros, 2012). 


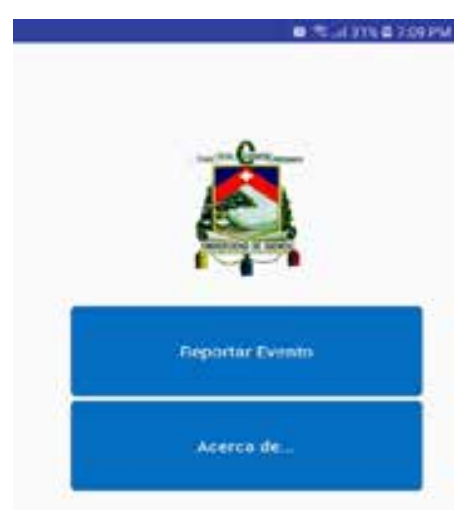

PPGIS Ucuenca
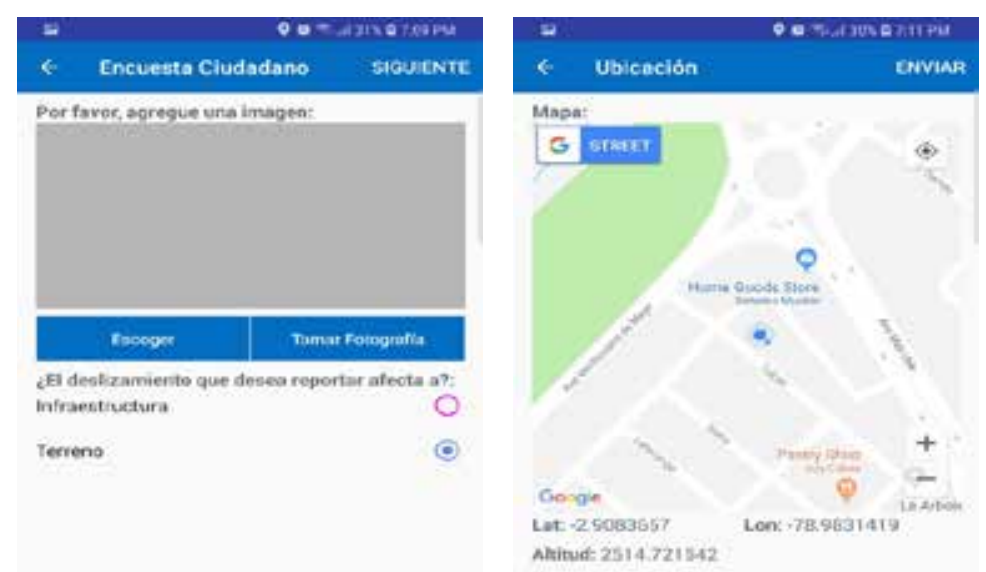

Figura 2. Capturas de la APP móvil

Por otra parte, la APP móvil cuenta con un sistema capaz de actualizar los formularios de manera remota, esto debido a que está programada para interpretar un archivo en formato JSON, que contiene los parámetros de la encuesta construida desde la plataforma IDE Ucuenca, para transformarlo en formularios con elementos HTML. De esta manera, la información de los formularios va a estar almacenada en el servidor de la IDE UCuenca y las actualizaciones se realizarán desde aquel sitio, sin la necesidad de actualizar la estructura desarrollada de manera nativa en la APP móvil (Figura 1).

La encuesta que reporte el usuario va a ser emitida hacia el servidor de la IDE UCuenca, para cumplir con un proceso de clasificación, almacenamiento en una Base de Datos espacial PostGIS y publicación de la información en el servidor de mapas GeoServer. Al contar esta información con un formato georreferenciado (Shapefile) es posible utilizarla para realizar un análisis que permita tomar decisiones para confrontar una situación. Entre las decisiones que se pueden tomar, están las de realizar una evaluación de la zona con equipos que puedan dar más detalle de la situación.

\section{EQUIPOS TECNOLÓGICOS Y LEVANTAMIENTO DE INFORMACIÓN}

\section{EQUIPOS TECNOLÓGICOS}

La Universidad de Cuenca ejecuta diversos proyectos de investigación enfocado al análisis geográfico, por los cuales han servido de exigencia para la adquisición de equipos como: El FARO Laser Scanner Focus3D (LIDAR) y el drone eBee (AG y RTK), que son tecnologías con características y funcionalidades diferentes, pero cuyos resultados pueden ser complementarios entre el uno y el otro. 


\section{FARO Laser Scanner Focus3D}

El Focus3D es un escáner láser 3D de alta velocidad para documentar y medir en forma detallada. El Focus3D utiliza tecnología láser para crear imágenes tridimensionales extraordinariamente detalladas de entornos complejos y geometrías de gran escala en tan solo algunos minutos (FARO, 2013).

\section{Drone eBee (AG y RTK)}

El senseFly eBee es un dron de mapeo completamente autónomo y fácil de usar. Su uso está enfocado principalmente para capturar fotos aéreas de alta resolución que puede transformar en ortomosaicos precisos y modelos 3D. El eBee puede cubrir hasta $12 \mathrm{~km} 2$ $(4,6 \mathrm{mi} 2)$ en un solo vuelo de mapeo automatizado, mientras que los vuelos sobre áreas más pequeñas, a altitudes más bajas, pueden adquirir imágenes con una distancia de muestreo de tierra de hasta $1.5 \mathrm{~cm}$ (0.6 in) por píxel (Sensefly, 2018).

\section{LEVANTAMIENTO DE LA INFORMACIÓN}

Partiendo delainformaciónque se ha obtenido mediantelaAPPmóvil(2.1), se puede determinar mediante una discusión y tomando en cuenta diferentes factores, si es factible o no realizar un análisis detallado de la zona, dando paso al despliegue del personal y el equipamiento (Drone y LIDAR) para realizar los estudios necesarios y proceder al levantamiento de la información.

Debido a que el Cantón Cuenca, perteneciente a la provincia del Azuay, se encuentra en una zona topográfica con problemas de erosión y terrenos inestables, la Municipalidad y su departamento técnico detectaron 60 lugares en los que se producen derrumbes y la destrucción de más de un centenar de viviendas (Telégrafo, 2012). Por lo tanto, este proyecto se ha centrado en evaluar algunas zonas que principalmente están activas con efectos de deslizamientos, estas zonas están situadas a los alrededores del cantón mencionado, entre ellas están: El Sector de Reina del Cisne, Trigales Altos y el sector de la Universidad de Azuay. Cada una de estas zonas se encuentran en una situación de riesgo, debido a que éstas abarcan a viviendas que han sido o están siendo afectadas de manera directa por los deslizamientos de terreno.

En esta sección del documento se va a exponer la metodología del levantamiento de la información en la zona del sector de la Universidad del Azuay, que puede ser ubicado geográficamente en las coordenadas: Latitud. -2.919889, Longitud. -79.001139. El estudio se inició el 06 de diciembre del 2017 y culminó el 20 de junio del 2018. Se compone de tres vuelos realizados con el drone eBee y un monitoreo utilizando el escáner láser terrestre FARO 3D X130 el 6 de diciembre del 2017.

\section{Levantamiento de información con el Escáner Láser}

Esta tecnología tiene un alcance de $130 \mathrm{~m}$ con una precisión de $+-2 \mathrm{~mm}$, dispara un láser infrarrojo en forma de onda y calcula la distancia a los objetos en función del desfase de la onda al momento del disparo y llegada al sensor. Esto es ideal para el estudio de zonas pequeñas (Revuelto, y otros, 2013), debido a que se debe dedicar una jornada extensa en la ubicación del LiDAR y sus esferas. 
Se efectuaron 15 escaneos tridimensionales con el escáner LiDAR el seis de diciembre del 2017, en una zona montañosa hacia al norte de la autopista Cuenca - Azogues, y el siete de diciembre del 2017 se realizaron 12 escaneos hacia la zona sur de la autopista correspondiente a la Universidad del Azuay.

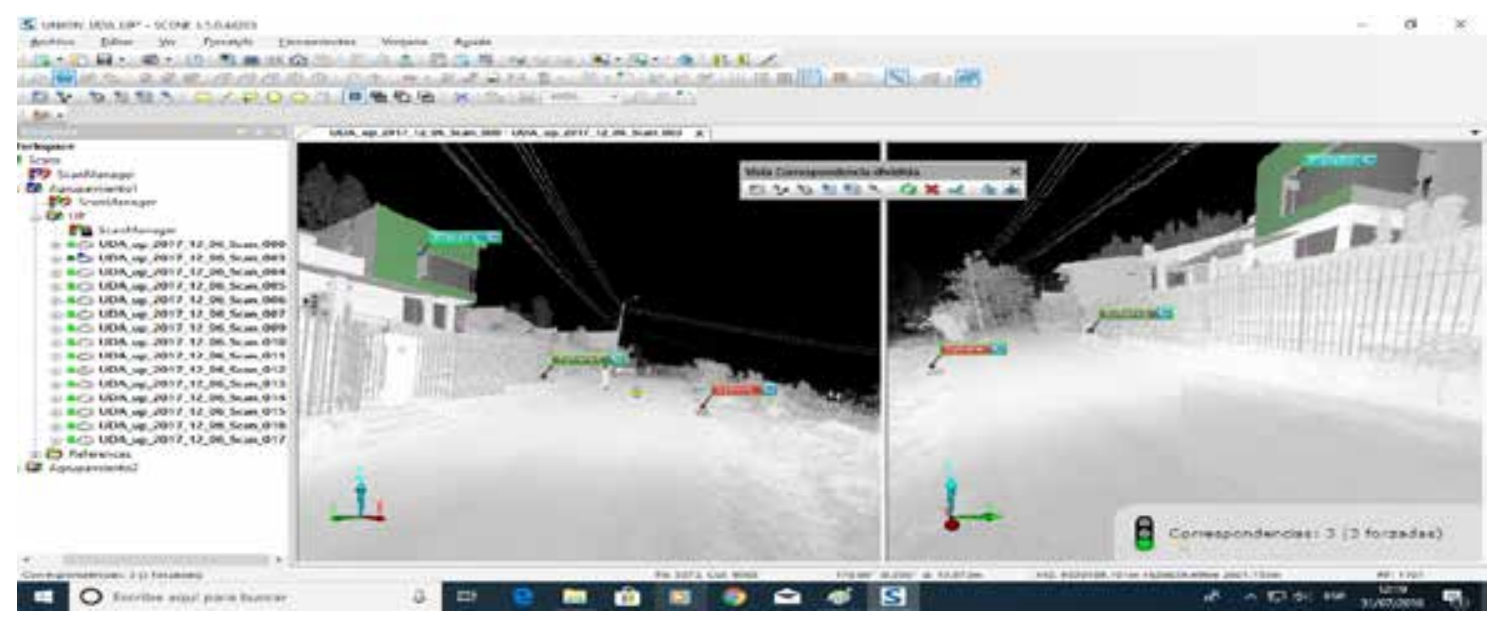

Figura 3. Unión de nube de puntos del LiDAR utilizado el Software FARO SCENE.

Los 27 levantamientos realizados por el escáner LiDAR terrestre de la zona norte y sur a la autopista, fueron unidos mediante el software FARO SCENE (Figura 3), utilizando esferas de $0.0698 \mathrm{~m}$ y $0.12335 \mathrm{~m}$ de radio, seis esferas como máximo para cada uno de los levantamientos (tres de vista frontal y posterior) y en algunos casos se utilizaron planos para disminuir el error de unión. Los escaneos realizados se ejecutaron en diferentes días; 15 de ellos fueron levantamientos en la parte superior, y los 12 restantes en la parte inferior; el procesamiento de la información se realiza de manera individual dado que son proyectos diferentes, pero la unificación de todos los escaneos se hizo posible gracias al software CloudCompare.

\section{Levantamiento de información con el drone eBee}

Los tres vuelos con el drone eBee se realizaron el 2 de febrero, 18 de abril y 20 de junio del 2018. Este drone cubrió $1.0618 \mathrm{~km} 2$ en un tiempo aproximado de 35 minutos para recorrer el área. El vuelo del mes de abril se realizó con un tamaño de pixel de $11 \mathrm{~cm}$, el cual sirvió para obtener el DSM (Modelo Digital de Superficie) que se utilizó para referenciar las elevaciones superficiales que compone el terreno, y así realizar vuelos posteriores con un tamaño de pixel más pequeño para obtener información más detallada. Adicionalmente se realizó un geoprocesamiento de la información para obtener el NDVI mediante el software ArcGIS 10.5.

Para un estudio más detallado, se obtuvieron ortofotos de la zona pertenecientes a cada año, que están a disposición en el Instituto Geográfico Militar (IGM), de los años 1963, 1977, 1979, 1994 y 2008. Con esto se obtuvo información para evaluar el deslizamiento por medio de una visión estereoscópica de esos años, en cuyas imágenes se aprecia la 
aparición de la cicatriz del escarpe y el hundimiento cercano a éste, en consecuencia, esta es la zona con mayor actividad de masa deslizada en la zona urbana del cantón Cuenca. Entre las jordanas realizadas durante el periodo de monitoreo de la zona de estudio, se realizaron levantamientos de información con estación total de puntos fijos y móviles en las fechas de diciembre del 2017, pero debido a los constantes mantenimientos de infraestructura que la Universidad del Azuay realiza en su terreno, se ubicaron nuevos puntos móviles en zonas más estratégicas en los meses de abril y junio del 2018.

Para monitorear el deslizamiento se realizaron dos vuelos con drone eBee en el mes de febrero y junio del 2018, con el fin realizar la comparación de nube de puntos; al ser un área muy grande, el drone es imprescindible para realizar una comparación de nube de puntos, pero tiene la desventaja de no ser muy precisa como otros instrumentos de medición (Escáner láser), por lo que se debe complementar con material topográfico (Carrullas \& Corominas J., 2017).

Una vez obtenida las fotografías del drone eBee, la información fue procesada mediante el software Agisoft PhotoScan Professional (febrero 2018) y Pix4D (junio 2018) (Figura 4). Estos programas computacionales trabajan con un algoritmo estándar llamado Structure From Motion (SFM), que realiza comparaciones cruzadas entre todas las fotografías, calculando los puntos comunes de forma similar a la fotogrametría estereográfica, pero con una matriz de imágenes para generar una nube de puntos (Tavani, y otros, 2014), en donde, entre los principales resultados se pudo obtener un modelo digital de elevación y un ortomosaico de la zona correspondiente.

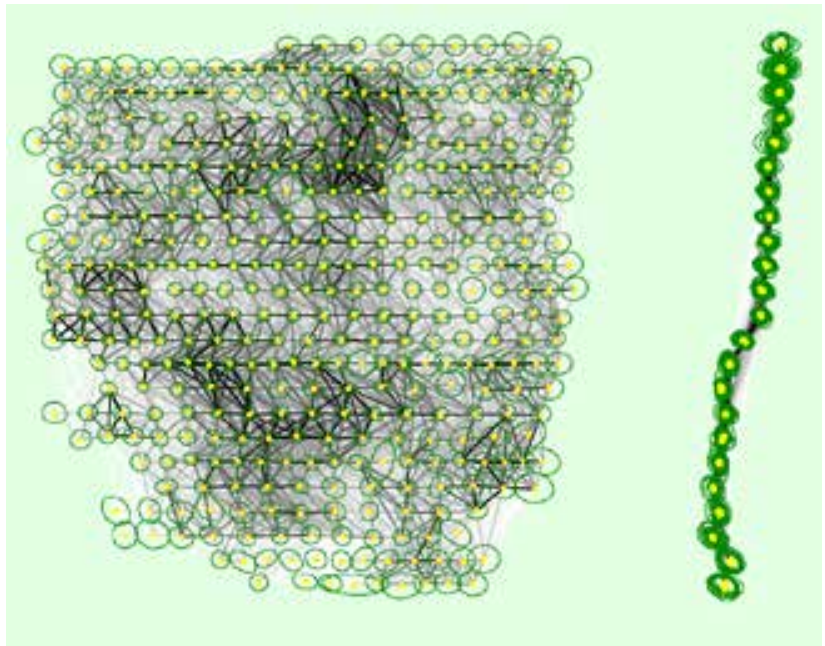

Figura 4. Unión de puntos de control con el software Pix4D.

\section{Estudios Adicionales}

Utilizando el software CloudCompare se realizaron geoprocesos para la unión de las nubes de puntos con el fin de obtener sus perfiles correspondientes. Se realizó la comparación entre la nube de puntos generada por el LiDAR terrestre en diciembre del 2017 y la nube 
de puntos generada por el drone de febrero del 2018 y una comparación entre las nubes de puntos generadas por el drone en los meses de febrero y junio del 2018.

Mediante el software AutoCAD Civil 3D se creó la representación del perfil de la zona de estudio de cada fecha el cual fue dividido en dos partes para un análisis más detallado debido a la extensa superficie de la zona.

Se tomaron dos muestras de suelo en la zona; la primera ubicada en la parte superior del deslizamiento y la otra cercana al río Yanuncay para realizar estudios de granulometría, expansividad y límites de atterberg. Para ampliar el conocimiento sobre qué material se encuentra bajo tierra se hizo una Tomografía de Resistividad Eléctrica con los equipos otorgados por la Red Sísmica del Austro de la Universidad de Cuenca.

Proyección de puntos al eje "Z”" (2d a 3d)

Una de las deficiencias que se puede encontrar en la APP móvil es la de obtener coordenadas con alta precisión; esto principalmente depende de la tecnología GPS (Global Positioning System) que dispone el dispositivo móvil, y el tiempo de espera para obtener la ubicación. los dispositivos antiguos pueden tener un margen de error de hasta 50 metros de exactitud (Cid F. P., 2017). Por otra parte, en la actualidad los nuevos dispositivos han mejorado notablemente su eficiencia con respecto a la precisión de la posición, reduciendo el margen de error de hasta 30 centímetros (Cid M. , 2017).

Parte de la información que emite la APP móvil no cuenta con la coordenada en el eje Z (Altitud), ya que en ciertas ocasiones las coordenadas se obtienen mediante la Operadora Móvil (No provee la altitud o no es aproximada) y no desde el dispositivo GPS. Debido a este inconveniente se ha realizado un proceso para proyectar cada punto reportado por la APP móvil hacia el eje Z, tal como se puede observar en la Figura 5, esto utilizando aplicaciones GIS de escritorio como ArcGIS.

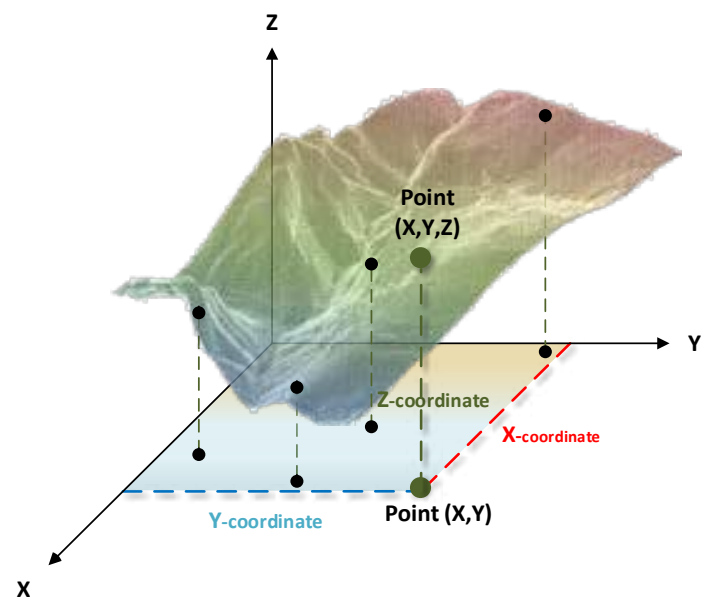

Figura 5. Proyección de puntos al eje Z. 


\section{PREPARACIÓN DE LA INFORMACIÓN}

Para llevar a cabo este objetivo se utilizó las herramientas ArcMap y ArcScene, la primera con el fin de realizar todos los geoprocesos necesarios para proyectar los puntos que se encuentran en el eje X, Y hacia el eje Z, y la segunda principalmente para visualizar la información con una perspectiva en 3D. Adicionalmente se utilizó un orto mosaico en RGB obtenida por el dron eBee, con la finalidad de visualizar los resultados obtenidos de una manera que se aproxime a la realidad.

En este proceso se llevó a cabo un procedimiento que utiliza el DEM (Modelo Digital de Elevaciones) de la zona de estudio, obtenido de manera gratuita desde el sitio WEB EarthExplorer de la USGS, y otro DEM obtenido desde un geoprocesamiento por parte del software PIX4D a partir del levantamiento de la información del drone eBee. Adicionalmente se obtuvo el Orto mosaico en RGB.

\section{MODELO DIGITAL DE ELEVACIONES Y GEOPROCESAMIENTO DE LA INFORMACIÓN}

Proporcionar esta elevación nos permite que la ortofoto se acomode al modelo de elevación con la finalidad de aproximarla a la forma del terreno real. Como resultado se obtiene las elevaciones de la ortofoto de la zona de estudio. En este proyecto el procedimiento mencionado se realizó para todas las zonas de estudio, pero en esta sección del documento se expone los resultados de la zona de Trigales Altos, que puede ser ubicado geográficamente en las coordenadas: Lat: -2.870294, Lon: -78.990772.

El motivo de utilizar información de diferente procedencia, tiene como fin observar cual es el grado de precisión o el margen de error que se puede obtener cuando se trabaja con información obtenida por un Satélite y un drone. Como ejemplo se puede observar en la Figura 6 un DEM obtenido desde el sitio WEB EarthExplorer y un drone eBee $(3 \mathrm{~cm} /$ px). De éstos se han obtenido las curvas de nivel con un intervalo de $5 \mathrm{~m}$ y notoriamente se puede observar que existe una diferencia en la transición de colores e intervalos que representan las elevaciones del terreno.

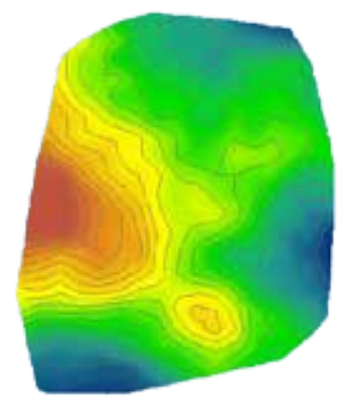

DEM EarthExplorer

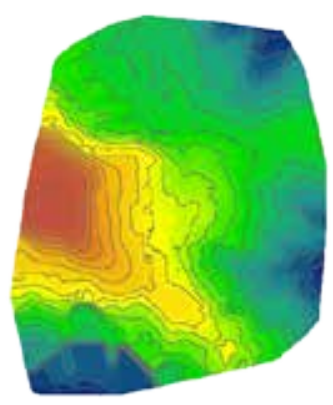

DEM drone eBee

Figura 6. DEM y curvas de nivel obtenido por EarthExplorer y el drone eBee. 
En la Tabla 1 se pueden observar parámetros que claramente pueden reflejar la diferencia entre un DEM y el otro, dando como resultado el nivel de detalle que existe entre ellos.

Tabla 1. Comparación de parámetros entre el DEM de EarthExplorer y el drone eBee.

\begin{tabular}{|c|l|r|r|}
\hline Tipo & Descripción & DEM EarthExplorer & DEM drone eBee \\
\hline \multirow{2}{*}{ DEM } & Filas y columnas & $17 \times 20$ & $2862 \times 3451$ \\
\cline { 2 - 4 } & Tamaño de celda & $30,81172816 \times 30,81172816$ & $0,17316 \times 0,17316$ \\
\hline \multirow{2}{*}{$\begin{array}{c}\text { Curvas } \\
\text { de nivel }\end{array}$} & Nro. de contornos & 39 & 335 \\
\cline { 2 - 4 } & Altura mínima & 2565 & 2600 \\
\cline { 2 - 4 } & Altura máxima & 2665 & 2690 \\
\hline
\end{tabular}

Para tener una perspectiva de la elevación terrestre en las zonas de estudio, se utilizó la opción Properties (Propiedades de la Capa) > Base Heights $>$ Floating on a custom superfice, y se seleccionó el modelo digital de elevaciones correspondiente. Este procedimiento se realizó tanto para el DEM, Ortomosaico RGB y los puntos reportados por la APP móvil. Este resultado puede ser apreciado tanto en la Figura 7 y Figura 8.
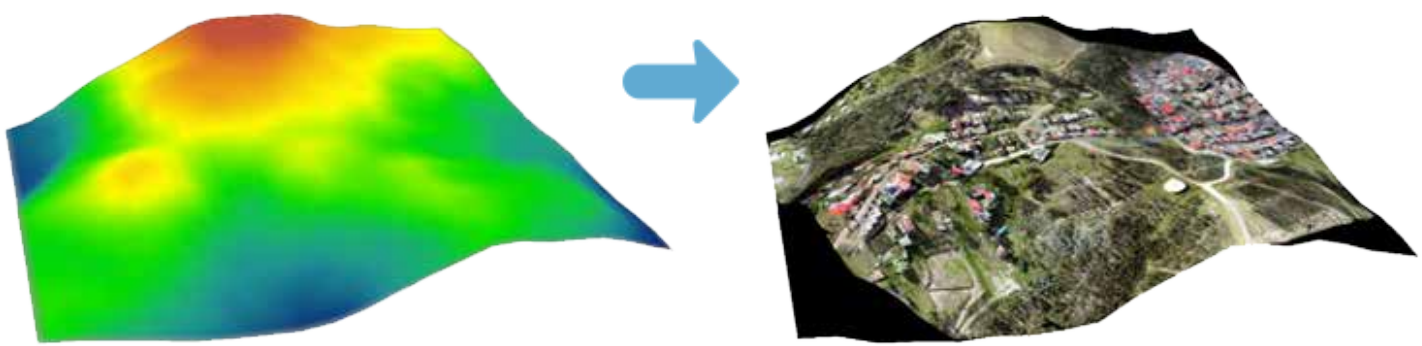

Figura 7 DEM Obtenido por el EarthExplorer.

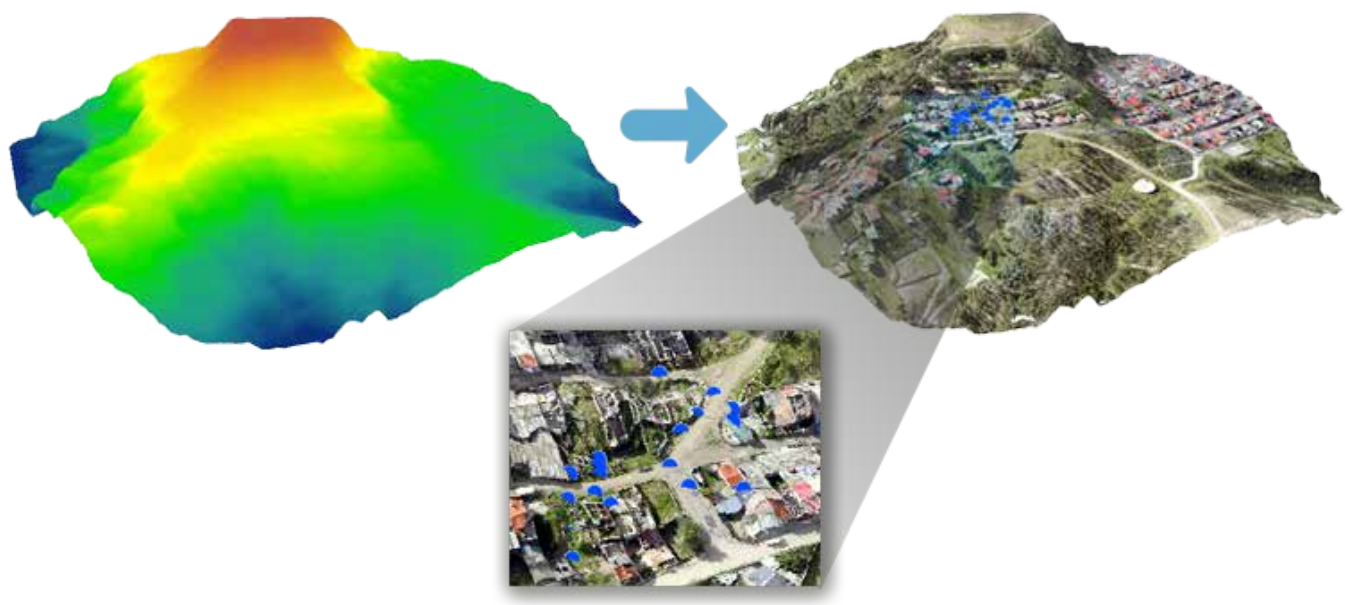

Figura 8. DEM Obtenido por el drone junto con la Interpolación de Puntos. 
Se puede observar como la Figura 7 muestra un terreno diferente a la Figura 8, que ha sido obtenida con un drone de alta precisión.

La Figura 8 expone las imágenes que representan el DEM, la Ortofoto con un modelo de elevaciones y los puntos proyectados en el eje $Z$. Para obtener los valores de los puntos en el eje $\mathrm{Z}$ se realizaron ciertos procedimientos utilizando la herramienta ArcScene.

En este proceso se utilizó la herramienta 3D Analyst $>$ Functional Surface $>$ Interpolate Shape de ArcScene.

Adicionalmente para obtener la coordenada $Z$ en la tabla de atributos de la capa, se utilizó la herramienta XTools Pro $>$ Table Operations > Add XYZ Coordinaties

Como resultado se ha observado que los valores en el eje $\mathrm{Z}$ proyectados por el DEM obtenido por EarthExplorer y el drone eBee varían parcialmente, en algunos casos hasta 34 metros de margen de error, esto debido a la escala con la que está representada la información; exponiendo de esta manera la precisión que puede ofrecer un drone en comparación con imágenes geográficas que se pueden encontrar en la WEB. Algunos de los resultados se dan a conocer en la Tabla 2. Esto motiva a que un usuario pueda preferir la información generada por el drone asegurando una mejor precisión para la evaluación de deslizamientos y movimientos de masa.

La información que recepta el Servidor de la IDE UCuenca mediante la APP móvil tiene diferentes objetivos, y uno de ellos es visualizar la información en una perspectiva de tres dimensiones, con la finalidad de complementar la información que se ha levantado mediante los equipos como el drone y Escáner Láser. En la Figura 9 se puede observar los puntos reportados por la APP móvil posicionados geográficamente en el eje $\mathrm{X}, \mathrm{Y}, \mathrm{Z}$, del visor $3 \mathrm{~d}$ de la plataforma IDE UCuenca.

\section{VISUALIZADOR DE NUBE DE PUNTOS WEB}

De manera constante la Infraestructura de Datos Espaciales (IDE) de la Universidad de Cuenca ha tratado de ofrecer a los usuarios tecnologías que puedan ser capaces de visualizar o analizar diferentes tipos de información geográfica. Actualmente las tecnologías GIS (Geographic Information System) pueden brindar diferentes perspectivas para el análisis de las dificultades que se pueden encontrar en un territorio determinado, una de estas perspectivas es la visualización tridimensional (3D).

$\mathrm{Al}$ contar con una nube de puntos factible para ser utilizada en el visualizador 3D, existe la posibilidad de publicar esta información a nivel de la WEB (Figura 9), obteniéndose la gran ventaja de acceder y utilizar esta información mediante un navegador, en cualquier instante o lugar. 
Una de las ventajas de esta herramienta, está principalmente la posibilidad de compartir un servicio WEB de la nube de puntos para que sea consumida por un cliente que utilice la misma tecnología, haciendo posible la integración de información adicional disponible en otro servidor.

Tabla 2. Comparación de resultados del eje Z con el DEM de EarthExplorer y drone eBee.

\begin{tabular}{|c|c|c|c|c|c|}
\hline \multicolumn{3}{|c|}{ DEM EarthExplorer } & \multicolumn{3}{|c|}{ DEM drone eBee } \\
\hline Eje X & Eje $Y$ & Eje Z & Eje X & Eje Y & Eje Z \\
\hline 723374,745 & 9682514,9 & 2622,731173 & 723374,745 & 9682514,9 & 2653,78225 \\
\hline 723387,551 & 9682471,71 & 2618,19061 & 723387,551 & 9682471,71 & 2651,750129 \\
\hline 723381,931 & 9682530,21 & 2622,176481 & 723381,931 & 9682530,21 & 2656,777288 \\
\hline 723345,752 & 9682543,93 & 2625,520684 & 723345,752 & 9682543,93 & 2657,370627 \\
\hline 723344,471 & 9682531,53 & 2626,361987 & 723344,471 & 9682531,53 & 2655,707108 \\
\hline 723339,346 & 9682540,37 & 2627,63226 & 723339,346 & 9682540,37 & 2657,006863 \\
\hline 723374,745 & 9682514,9 & 2622,731173 & 723374,745 & 9682514,9 & 2653,78225 \\
\hline 723348,786 & 9682504,33 & 2628,922368 & 723348,786 & 9682504,33 & 2660,39539 \\
\hline 723349,767 & 9682523,61 & 2626,523087 & 723349,767 & 9682523,61 & 2655,272466 \\
\hline 723328,26 & 9682525,25 & 2633,177918 & 723328,26 & 9682525,25 & 2659,464655 \\
\hline 723328,26 & 9682525,25 & 2633,177918 & 723328,26 & 9682525,25 & 2659,464655 \\
\hline 723364,627 & 9682513,01 & 2624,79075 & 723364,627 & 9682513,01 & 2654,583539 \\
\hline 723374,024 & 9682489,23 & 2622,356047 & 723374,024 & 9682489,23 & 2654,009856 \\
\hline
\end{tabular}

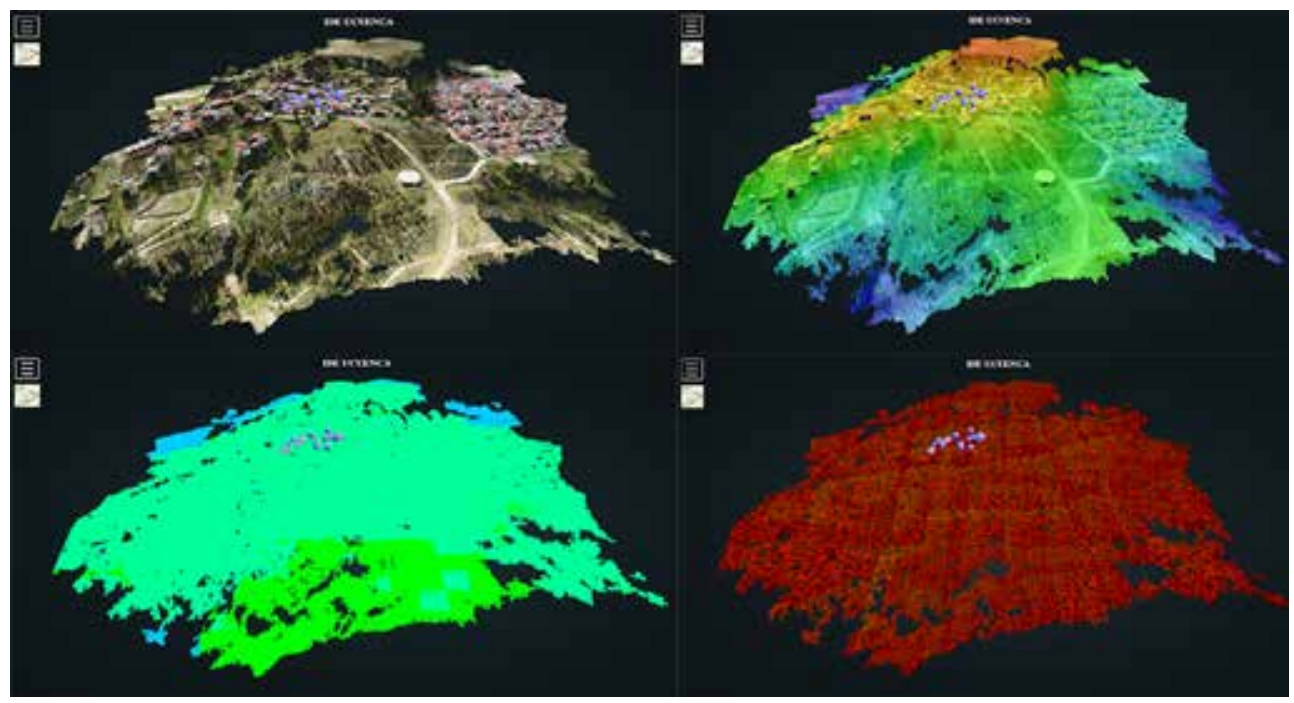

Figura 9. Capturas del visualizador de nube de puntos 


\section{CONCLUSIONES}

Con los resultados obtenido se puede concluir los siguiente:

- A partir del uso de una APP móvil se puede obtener información geográfica capaz contribuir al análisis de una superficie terrestre, y mediante ello a la toma de decisiones.

- La precisión obtenida por el drone eBee se acerca mucho más a la realidad en comparación de imágenes satelitales, ya que el nivel de detalle de la información es formidable, evidencia las ventajas para determinar movimientos en masa por comparación de nube de puntos en tomas realizadas en tiempos diferentes.

- El visualizador 3D permite a los usuarios visualizar, medir y anotar cualquier nube de puntos desde un simple navegador WEB.

- La combinación de diferentes tecnologías contribuye a la obtención de diferente tipo de información geográfica y al análisis en diferentes perspectivas.

\section{TRABAJOS FUTUROS}

- Es necesario determinar con mayor precisión los niveles mínimos de determinación de movimientos en masa, se está trabajando en una metodología para conseguir mayor precisión utilizando RTK en los vuelos.

- El visualizador 3D aún puede tener mayores funcionalidades, en la medida que se permita el análisis de información. Por ahora la integración de nube de puntos es manual siguiendo un tutorial, sin embargo, así como ya se ha logrado la incorporación de herramientas para la integración de imágenes ráster en el IDE UCuenca, se está trabajando en la incorporación de herramienta similar para el visor 3D. Esto permitirá a un usuario no especializado fácilmente integrar la nube de puntos propia y visualizarla.

\section{AGRADECIMIENTOS}

Este proyecto es financiado por los fondos DIUC bajo el XV concurso de proyectos, con el nombre "Geoprocesamiento IDE como apoyo al análisis de vulnerabilidades y planificación territorial" 2017-2018. Especial agradecimiento al Ing. Ricardo Peñaherrera e Ing. Leonardo Espinosa de la Secretaria de Gestión de Riesgos por su apoyo para la colaboración de dicha entidad en lo referente a alertas tempranas. Al GT-IDE (Grupo de Trabajo IDE) de CEDIA por su constante apoyo en temas relacionados a las Infraestructura de Datos Espaciales.

\section{BIBLIOGRAFÍA}

Brown, G. (Junary de 2012). Public Participation GIS (PPGIS) fro Regional and Environmental Planning: Reflections on a Decade of Emprical Research. Urisa Journal, 25(2):5-16.

Carrullas, J., \& Corominas J., H. M. (2017). Experiencias con drones para el estudio de movimientos de ladera. IX Simposio Nacional sobre Taludes y Laderas Inestables.

Cid, F. P. (Junio de 2017). Comparativa de métodos de Localización con Smartphones. Barcelona. 
Cid, M. (22 de 09 de 2017). XtakaMóvil. Obtenido de https://www.xatakamovil.com: https:// www.xatakamovil.com/movil-y-sociedad/el-gps-de-tu-proximo-movil-sera-mucho-maspreciso-solo-30-centimetros-de-margen-de-error

FARO. (10 de 2013). Runco. Obtenido de http://www.runco.com.ar/IMG/pdf/FARO_Focus3D-S_ SPA_pdf.

Hahmann, S., \& Burghardt, D. (23 de Nov de 2012). How much information is geospatially referenced? Networks and cognition. International Journal of Geographical Information Science.

Olaya, V. (2014). Sistemas de Información Geográfica (Vol. 2).

Revuelto, J., López-Moreno, J. I., Azorín-Molina, C., Arguedas, G., Vicente-Serrano, S. M., \& Serreta, A. (2013). Application of terrestrial laser scanner techniques for monitoring dynamic geomorphological processes: snow accumulation and ice masses in mountain areas. Cuadernos de Investigación Geográfica. doi:https://doi.org/10.18172/cig.1994

Sensefly. (2018). Sensefly. Obtenido de https://www.sensefly.com/drone/ebee-mapping-drone/.

Sequeiros, C. (30 de Oct de 2012). Teoría y Práctica de la Encuesta en Árbol. VIII Congreso Nacional de Sociología, 2-3.

Tavani, S., Granado, P., Corradetti, A., Girundo, M., Iannace, A., Arbués, P., . . Mazzoli, S. (February de 2014). Building a virtual outcrop, extracting geological information from it, and sharing the results in Google Earth via OpenPlot and Photoscan: An example from the Khaviz Anticline (Iran). Computers \& Geosciences, 63. doi:https://doi.org/10.1016/j. cageo.2013.10.013

Telégrafo, E. (18 de 06 de 2012). https://www.eltelegrafo.com.ec. Obtenido de https:// www.eltelegrafo.com.ec/noticias/2012/1/cuenca-tiene-60-sitios-en-riesgo-por-losdeslizamientos. 